\title{
Conjugated polymer nanoparticles
}

\author{
Dönüs Tuncel ${ }^{* a c}$ and Hilmi Volkan Demir ${ }^{b c}$ \\ Received (in Zürich, Switzerland) 29th November 2009, Accepted 24th January 2010 \\ First published as an Advance Article on the web 6th March 2010 \\ DOI: 10.1039/b9nr00374f
}

Conjugated polymer nanoparticles are highly versatile nano-structured materials that can potentially find applications in various areas such as optoelectronics, photonics, bio-imaging, bio-sensing and nanomedicine. Their straightforward synthesis in desired sizes and properties, biocompatibility and non-toxicity make these materials highly attractive for the aforementioned applications. This feature article reviews the recent developments in the synthesis, characterization, properties and application of these exciting nanostructured materials.

\section{Introduction}

Energy and healthcare are two important areas for human beings and are directly related to our well-being. Every step attempted towards resolving energy and health related issues is highly vital. To this end, new methods and materials for energy generation

${ }^{a}$ Deparment of Chemistry, Bilkent University, Bilkent, Ankara, Turkey 06800.E-mail: dtuncel@fen.bilkent.edu.tr

${ }^{b}$ Department of Physics, Department of Electrical and Electronics Engineering, Nanotechnology Research Center, Bilkent, Ankara, Turkey, 06800

'Institute of Materials Science and Nanotechnology, Bilkent University, Bilkent, Ankara, Turkey 06800 and saving as well as early diagnosis of diseases using easy and cheap tools are indispensable. Among them, nanoparticles based on conjugated polymers are emerging as multifunctional nanoscale materials that promise great potential to offer exciting opportunities in these areas (e.g., as imaging agent, biosensor, photonic and optoelectronic device materials). These conjugated polymer nanoparticles (CPNs) are desirable for a number of reasons. Their properties can be tuned easily for desired applications through the choice of conjugated polymers and surface modification. Additionally, their easy synthesis, tuneable properties and less toxicity and more biocompatibility comparedto the existing inorganic nanoparticles further make these materials highly attractive in the material choice. This feature article

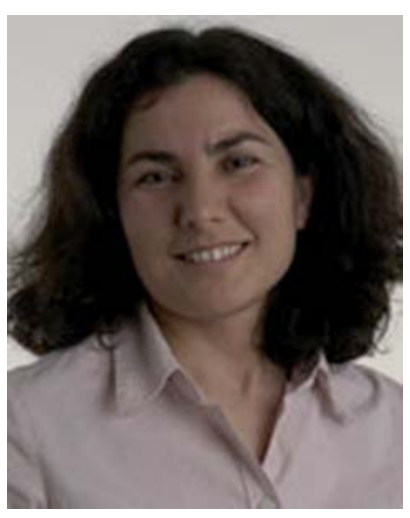

Dönüs Tuncel
Dönüs Tuncel received her BSc in Chemistry from University of North London. She went on to study for her PhD at Cambridge University in Melville Polymer Synthesis Laboratory; after a while she moved to Imperial College, London and completed her study in 1999 under the supervision of Dr Joachim Steinke in the area of polymer synthesis and supramolecular chemistry. After working as a postdoctoral research assistant for 3 years in the Chemistry Department of Oxford University under the direction of Professor Harry L. Anderson, she took a position in 2003 as Assistant Professor in the Chemistry Department of Bilkent University, Ankara. Her research combines synthetic organic, supramolecular and polymer chemistry to prepare functional materials such as supramolecular polymers, cucurbituril containing rotaxanes and polyrotaxanes, water soluble conjugated polymers, functional and mechanically-stable conjugated polymer nanoparticles which have potential applications in the use of polymeric opto-electronic devices (LEDs, solid state lighting and photovoltaic devices), chemo- and bio-sensors, and molecular switches.

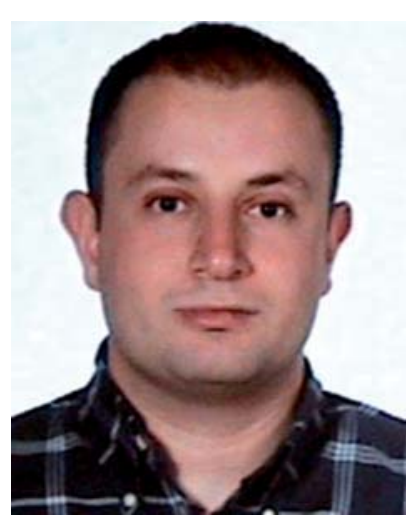

Hilmi Volkan Demir received his BSc degree in electrical and electronics engineering from Bilkent University, Ankara, Turkey, in 1998, and his MS and PhD degrees in electrical engineering from Stanford University, CA, USA, in 2000 and 2004, respectively. He received his Docent title (assoc. prof.) in optics and photonics from Turkish Council of Higher Education in 2007. Since 2004, he has been working as a faculty member at Bilkent University, with joint appointments at the Department of Physics and the Department of Electrical and Electronics Engineering, where he also serves as the Founding Director of the Devices and Sensors Laboratory, Associate Director of Nanotechnology Research Center, and a faculty member of Institute of Materials Science and Nanotechnology. His research interests include the study of lightmatter interactions at nanoscale for a variety of nanomaterials and the development of nanoparticle/nanostructure embedded devices (including solid-state lighting devices, photovoltaic devices, modulators, and switches). He is a winner of the European Science Foundation European Young Investigator (ESF-EURYI) Award. 
focuses on these water-dispersible conjugated polymer nanoparticles, and its purpose is to give an overview of the main research works conducted relating to these nanoparticles by discussing their synthetic methods, their properties and to provide some examples to demonstrate their applications. The article also discusses briefly their shortcomings and foreseen problems that should be solved before they could be available commercially.

\section{Current methods to prepare conjugated polymer nanoparticles}

CPNs are prepared mainly by miniemulsion and reprecipitation methods. In the following section we are going to discuss each method by emphasizing each method's advantages and disadvantages.

\subsection{Miniemulsion}

This is the most common method used in the synthesis of conjugated nanoparticles. Using this method illustrated in Fig. 1, Landfester and co-workers prepared nanoparticles from various polymers including conjugated polymers. ${ }^{1-4}$ To prepare CPNs, the polymer is dissolved in a water immiscible organic solvent and then the resulting solution is injected into an aqueous solution of an appropriate surfactant. ${ }^{5}$ The mixture is stirred rapidly by ultrasonicating to form stable miniemulsions containing small droplets of the polymer solution. The organic solvent is evaporated to obtain a stable dispersion of polymer nanoparticles in water. The size of nanoparticles could vary from $30 \mathrm{~nm}$ to $500 \mathrm{~nm}$ depending on the concentration of the polymer solution. However, the droplets could be destabilized by Ostwald ripening as well as the flocculation caused by the coalescence of droplets. To prevent flocculation appropriate surfactants are used, while Ostwald ripening can be suppressed by the addition of a hydrophobic agent (a hydrophobe) to the dispersed phase. The hydrophobe promotes the formation of an osmotic pressure inside the droplets that counteracts the Laplace pressure (the pressure difference between the inside and the outside of a droplet) preventing diffusion from one droplet to the surrounding aqueous medium.

In the literature most of the examples involve the preparation of nanoparticles from the preformed polymers using an oil-in-water system. However, Müllen et al. demonstrated the synthesis of nanoparticles starting from monomers in non-aqueous emulsions (oil-in-oil). ${ }^{6}$ In this example, cyclohexane as the continuous phase and acetonitrile as the dispersed phase were used while

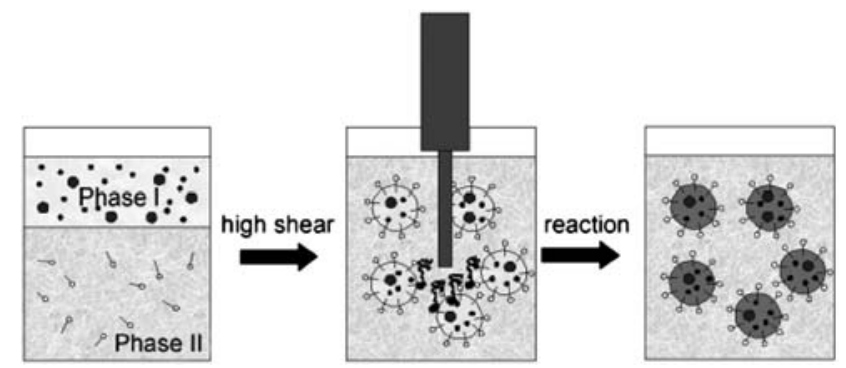

Fig. 1 The preparation of nanoparticles using the miniemulsion method. Reproduced with permission from ref. 1a (Copyright 2009 Wiley-VCH Verlag GmbH \& Co. KGaA). polyisoprene-block-poly(methyl methacrylate) (PI-b-PMMA) was utilized as an emulsifying agent. Poly(3,4-ethylenedioxythiophene) (PEDOT), polyacetylene and poly(thiphene3 -yl-acetic acid) were prepared as nanoparticles by catalytic and oxidative polymerization using this method. Moreover, after the formation of nanoparticles the emulsifying agent (PI-b-PMMA) was removed by washing off with THF. The number-average diameter of the particles is measured as $43 \mathrm{~nm}( \pm 10 \mathrm{~nm})$.

In 2006, Mecking and co-workers reported on the synthesis of the processable polyacetylene nanoparticles by the polymerization of acetylene in an aqueous miniemulsion method. ${ }^{7}$ For this purpose, a Pd catalyst was dissolved in a minimum amount of a mixture of hexane-ethanol, and added into an aqueous solution of surfactant and organic acid (sodium dodecylsulfate and methane sulfonic acid). Subsequently, this mixture was sonicated to form a miniemulsion. An intensely coloured, black dispersion of polyacetylene was obtained by stirring the miniemulsion under an acetylene atmosphere. The size of polyacetylene nanoparticles was determined as approximately $20 \mathrm{~nm}$ by transmission electron microscopy (TEM).

Very recently Mecking et al. demonstrated that the nanoparticles of poly(arylene diethynylene) (arylene $=2,5$-dialkyoxyphenylenes and 9,9'-dihexylfluorene) derivatives could also be directly prepared by miniemulsion polymerization of appropriate monomers under Glaser coupling conditions. ${ }^{8}$ The molecular weights of these nanoparticles were measured to be in the range of $M_{\mathrm{n}} 10^{4}$ to $10^{5} \mathrm{~g} \mathrm{~mol}^{-1}$ by gel permeation chromatography (GPC) and their sizes were determined to be around $30 \mathrm{~nm}$ by TEM. They also incorporated covalently $0.1-2 \mathrm{~mol} \%$ perylene dye and 2-9 $\mathrm{mol} \%$ fluorenone dye, respectively to the poly(arylene diethynylene) to obtain conjugated nanoparticles made from these co-polymers with the desired emission wavelength through energy transfer.

\subsection{Reprecipitation}

In this method ${ }^{9-17}$ presented in Fig. 2, a hydrophobic conjugated polymer is dissolved in a good solvent (e.g., THF) for the polymer and poured into a poor solvent (e.g., water), which is miscible with the good solvent. The resulting mixture is stirred vigorously usually using a sonicator to assist the formation of nanoparticles. After the nanoparticle formation the organic solvent is removed to leave behind water dispersible

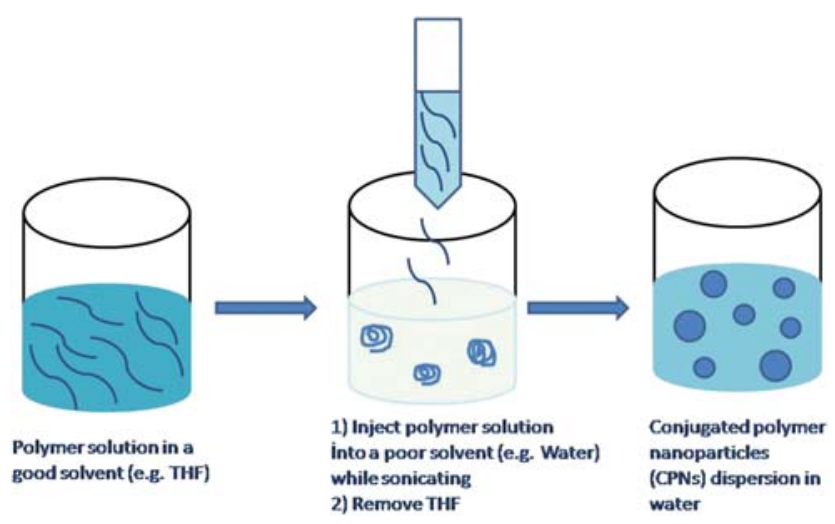

Fig. 2 Preparation of polymer nanoparticles by the reprecipitation method. 
nanoparticles. The main driving force for the formation of nanoparticles is the hydrophobic effect. When the solution of polymer in organic solvent is added to water, polymer chains tend to avoid contacting with water and consequently in order to achieve minimum exposure they fold into spherical shapes. The preparation does not involve the use of any additives such as surfactants and hydrophobes and can be applied to a wide variety of conjugated polymers that are soluble in organic solvents. Moreover, using this method, it is possible to tune the size of nanoparticles by adjusting the polymer concentration and using polymers with appropriate molecular weights. For example, nanoparticles are produced with 5-10 $\mathrm{nm}$ diameter containing single polymer chains.

Moon et al. reported the synthesis of nanoparticles from poly(phenylene ethynylene) (PPE) derivatives as shown in Fig. 3 by phase inversion precipitation. ${ }^{18}$ The synthesis involves the dissolution of the polymer in DMSO and the subsequent addition of this solution into an aqueous SSPE buffer (saline, sodium phosphate, EDTA). The electron micrographs revealed particles with diameters of 500-800 nm. Dynamic light scattering (DLS) results show a mean particle size of $400-500 \mathrm{~nm}$. The chemical structure of the polymer, especially the nature and density of the hydrophilic side groups, affected the particles formation. Apparently, the side chain with the protonated amine and the short chain nonionic diethylene oxide moiety stabilise the surface of the forming particle and hinder aggregation and precipitation.

The same group reported smaller nanoparticles (as small as $8 \mathrm{~nm}$ ) from similar hydrophilic conjugated polymers shown in Fig. 3 by tuning the variables in the particle formation process, which include $\mathrm{pH}$, the nature of the acid, salt concentration, and mixing parameters. ${ }^{19,20}$ In this work, the resulting nanoparticles were purified by sequential ultrafiltration with acetic acid or tartaric acid, ethylenediaminetetraacetic acid (EDTA), and water. Acid aided removal of metal-ion contamination (palladium and copper) and reduced aromatic backbone aggregation in the nanoparticle formation by generating repulsive forces.

\section{Photophysical properties of nanoparticles}

If CPNs are going to be utilized in the fabrication of optoelectronic devices, it is crucial to know whether the photophysical and optical properties of the polymers influence the nanoparticle formation and, if it is the case, to what extent. Moreover, CPNs prepared through the miniemulsion method contain some additives such as surfactants and hydrophobes so that it is again important to reveal the effects of these additives on the photophysical properties of the nanoparticles.

Piok et al. conducted a detailed study to reveal the photophysical properties of CPNs prepared by the miniemulsion method out of methyl-substituted ladder-type poly(para-phenylene) (m-LPPP). ${ }^{21}$ They studied the steady-state and nonequilibrium photoexcitation dynamics of CPNs and compared those results to the film of the same polymer of bulk solution. The results indicated that the processing of the m-LPPP into nanoparticles has not significantly altered its optical spectroscopic properties. Surprisingly, size-dependent changes were also not observed in the emission and the absorption spectra of CPNs having particle sizes ranging from 70 to $160 \mathrm{~nm}$ in diameter. The surfactant effect on the optical properties of CPNs was also investigated. For this purpose, a film was prepared from the bulk solution of the polymer in the presence of the surfactant and then this was compared with the film taken from the surfactant free polymer bulk solution. They did not observe any changes associated with the presence of the surfactant in the emission and the absorption spectra of the films. However, they observed significant differences in the photoexcitation kinetics and experimentally showed that the film of nanoparticles had an influence on the generation, migration and recombination behaviour of the photoexcited species.

Using the reprecipitation method it is possible to prepare nanoparticles in desired sizes down to the size of a single chain containing nanoparticles. Therefore, it would be very interesting and useful to reveal the size-dependent photophysical properties of these nanoparticles. However, we should emphasize that CPNs are different from the quantum dots which exhibit sizedependent photophysical properties due to quantum confinement effect, whereas the changes in the optical properties of CPNs are mainly dependent on the conformational changes of the polymers and the nature of the aggregates.

Masuhara and co-workers prepared nanoparticles with various sizes $(40-400 \mathrm{~nm})$ from poly(3-[2-(N-dodecylcarbamoyloxy)ethyl]thiophene-2,5-diyl) (P3DDUT) and investigated their

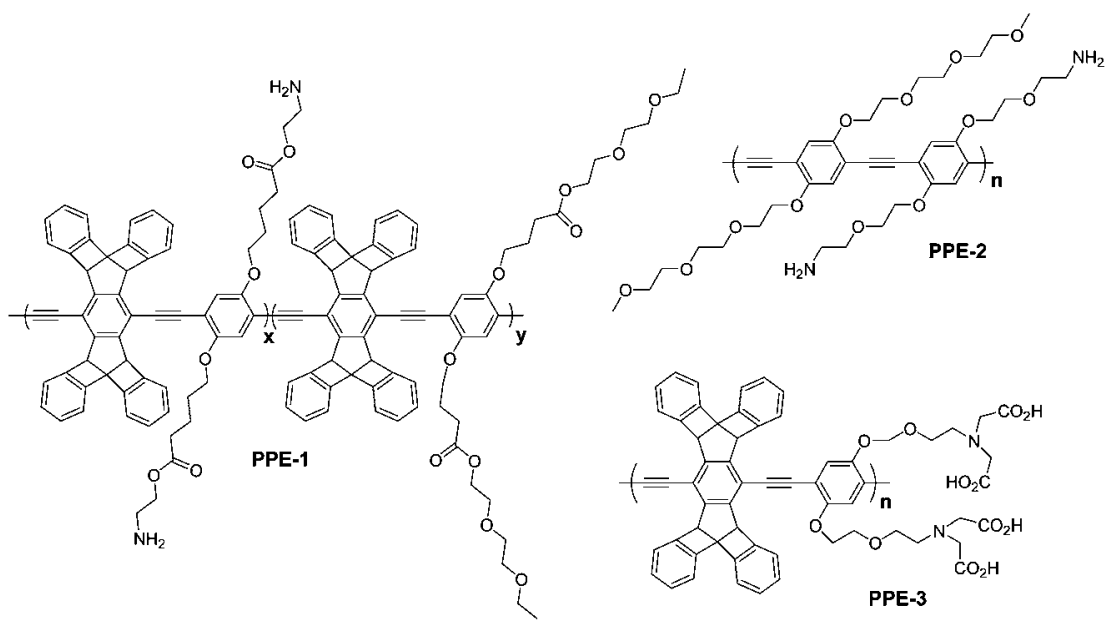

Fig. 3 The structures of poly(phenylene ethynylene) (PPE) derivatives utilized in the preparation of CPNs. 
spectroscopic and thermochromic behaviour in water and also made films from their water dispersions. ${ }^{17}$ They observed blue shifts in the absorption and emission spectra of nanoparticles upon decreasing their sizes from 400 to $40 \mathrm{~nm}$; this is in contrast to the studies of Pick et al. ${ }^{21}$ in which they reported no changes in the optical properties of the polymers upon nanoparticle formation.

Via the reprecipitation method, we prepared nanoparticles with sizes ranging from 5-70 $\mathrm{nm}$ by varying the concentration of poly(9,9-dihexylfluorene) (PDHF) solution and studied the sizedependent photophysical properties of these nanoparticles in dispersion and after forming films by drop-casting. In the emission spectrum, the peak of the $0-0$ electronic transition redshifted by a total of $33 \mathrm{~nm}$ going from bulk solution to a thin film; however, this red-shift was only around $11 \mathrm{~nm}$ for converting a dispersion of large nanoparticles into a film, and $13 \mathrm{~nm}$ in the case of a small nanoparticle film. The film of the nanoparticles with larger size distribution exhibited a fluorescent quantum yield of $43 \%$, while the fluorescent yield of the small nanoparticle film was calculated as $68 \%$, which surpassed their polymer thin films with values ranging from $23 \%$ to $44 \%$ depending on the film thickness. ${ }^{22}$

A detailed photophysical property investigation of nanoparticles prepared from poly[2-methoxy-5-(2-ethylhexyloxy)-1,4phenylenevinylene] (MEH-PPV) with different sizes in the range of 10-100 nm was conducted by Barbara et al. For this work, they used single-particle spectroscopy techniques, including both static and dynamic measurements and investigated the effect of particle size on the frequency-resolved photoluminescence spectra, the photobleaching dynamics, and triplet-triplet annihilation kinetics. They found out that the nanoparticles with diameter greater than $\sim 10 \mathrm{~nm}$, which contain more than one polymer chains, exhibited bulk-like structure and properties. They attributed these changes to the distance dependence of four main processes: electronic energy transfer between blue and red sites, triplet-triplet annihilation, singlet exciton quenching by triplets, and singlet exciton quenching by hole polarons. ${ }^{23,24}$

The dynamics and mechanisms of cellular processes can be studied by fluorescent labelling and detection of target biological molecules in live cells. The fluorescent labels that can be used for these applications should feature good photostability, high fluorescent quantum yield, biocompatibility and non-cytotoxicity. Small fluorescent dyes were used for these purposes but they exhibit poor photostability, which limits their use in longterm monitoring of live cells. Quantum dots have high extinction coefficient and photostability and thus can be utilized as fluorescent labels; however, the cytotoxicity is an important consideration in their usage. ${ }^{25,26}$ For this purpose, conjugated polymers are considered as suitable probes for fluorescence microscopic imaging by meeting these conditions required for an ideal fluorescent probe. Moreover, their structure can be tuned easily to obtain desired properties and functional groups can be incorporated to attach biological molecules. For instance, by using water-soluble conjugated polymers the detection of nucleic acids, proteins, bacteria, and cancer cells in vitro has been demonstrated. ${ }^{27-32}$

Conjugated polymer based nanoparticles represent a new class of highly fluorescent probes. They can be prepared practically from any conjugated polymers; they are water dispersible and show higher brightness and better photostability than molecular dyes because each nanoparticle contains a number of chromophores. They are also very promising candidates for two-photon (2P) imaging. In the $2 \mathrm{P}$ fluorescence imaging technique it is possible to image living tissues up to a depth of one millimetre and therefore this technique finds applications in many important areas such as neurobiology, immunology, and tissue engineering. ${ }^{33}$ To this end, McNeill and co-workers prepared nanoparticles from a series of hydrophobic conjugated polymers by the reprecipitation method to study the two-photon excited fluorescence properties and explore their application for multiphoton fluorescence microscopy. The measurements of the nonlinear optical properties of the CPNs indicate extraordinarily high two-photon absorption (TPA) cross-sections; for instance TPA was found in one case as high as $2.0 \times 10^{5} \mathrm{GM}(1 \mathrm{GM}$ $($ Goeppert-Mayer $)=10^{-50} \mathrm{~cm}^{4} \mathrm{~s}_{\text {photon }}{ }^{-1}$ molecule $\left.^{-1}\right)$, which is apparently the highest value reported to date for a particle of comparable size (Fig. 4). This 2P fluorescence of single nanoparticles was demonstrated using relatively low laser power, demonstrating the great potential of these CPNs for multiphoton fluorescence imaging applications. ${ }^{\mathbf{1 0}}$

In another study, McNeill et al. studied in details the photophysical properties of CPNs in order to determine their suitability as a fluorescent probe in the application of live cells imaging. ${ }^{11}$ The data gathered from the photophysical studies showed that these nanoparticles exhibit extraordinary fluorescence brightness coupled with excellent photostability (Table 1). Single particle fluorescence imaging and kinetic studies indicated much higher emission rates $\left(10^{8} \mathrm{~s}^{-1}\right)$ and little or no blinking of the nanoparticles as compared to typical results for single dye molecules and quantum dots. For example, the most photostable dyes such as Rhodamine $6 \mathrm{G}$ can emit only around $10^{6}$ photons (a)

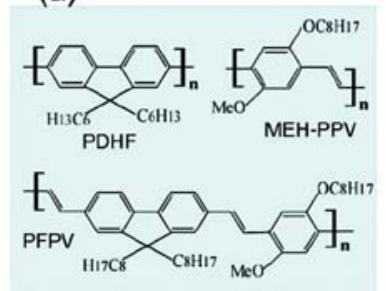

(b)

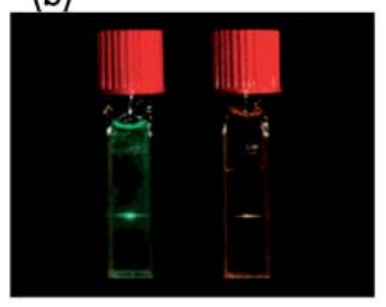

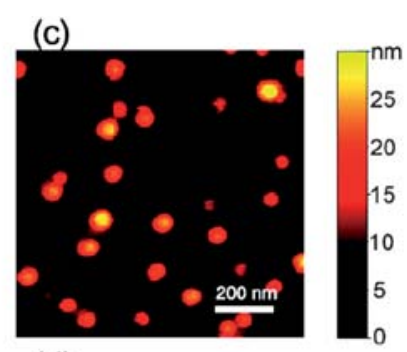

(d)

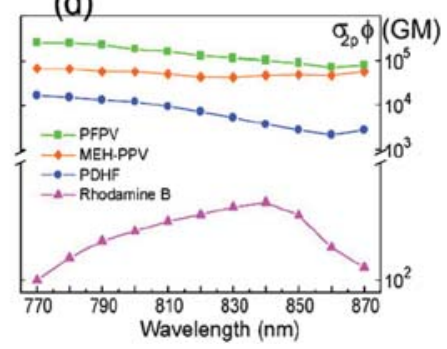

Fig. 4 (a) Chemical structures of the conjugated polymers. (b) Photograph of the fluorescence from aqueous CPN dispersions under two photon excitation of an $800 \mathrm{~nm}$ mode-locked Ti:sapphire laser. (c) A typical the atomic force microscopy (AFM) images of the PFPV dots on a silicon substrate. (d) Semilog plot of two-photon action cross sections versus the excitation wavelength for CPNs and rhodamine B (reference compound). Reprinted with permission from ref. 10 (Copyright 2007 The American Chemical Society). 
Table 1 Figures of merit evaluating the multicolor conjugated polymer dots as fluorescent tags. Reprinted with permission from ref. 11 (Copyright 2008 The American Chemical Society)

Cpdots (ave diameter $15 \mathrm{~nm}$ )

(absorption/fluorescence max, $\mathrm{nm}$ )

PFO (380/435)

PPE $(390 / 440)$
$4.6 \times 10^{-13}$
0.12
242
$5.0 \times 10^{8}$
$\sim 10^{-9}$
$\sim 10^{8}$

PFPV $(445 / 510)$
$5.5 \times 10^{-13}$
0.08
133
$6.0 \times 10^{8}$
$\sim 10^{-8}$
$\sim 10^{7}$

$5.4 \times 10^{-13}$
0.40
270
$1.5 \times 10^{9}$
$\sim 10^{-8}$
$\sim 10^{7}$

absorption cross section $\left(\sigma, \mathrm{cm}^{2}\right)$

quantum yield $\left(\Phi_{\mathrm{F}}\right)$

fluorescence lifetime $(\tau, \mathrm{ps})$

radiative rate $\left(k_{\mathrm{r}}, \mathrm{s}^{-1}\right)$

photobleaching quantum yield $\left(\Phi_{\mathrm{B}}\right)$

photo number (photons)

before irreversible photobleaching which is not an appropriate value for long-term single molecule fluorescence tracking. Analysis of single particle photobleaching trajectories revealed excellent photostability, as many as $10^{9}$ or more photons emitted per nanoparticle prior to irreversible photobleaching (Fig. 5). All these results clearly indicate that these CPNs are very promising for demanding fluorescence-based imaging and sensing applications such as high speed super-resolution single molecule/particle tracking and highly sensitive assays. ${ }^{11}$

\section{Energy transfer studies}

Fluorescence energy transfer (or Förster resonance energy transfer) (FRET) receives increasingly more attention owing to its potential applications in a number of areas such as molecular beacon biosensors and optoelectronic devices. In FRET, the excitation energy of the donor fluorophore is transferred to acceptor fluorophore through a nonradiative pathway. The transfer rate depends on the inverse sixth power of the distance
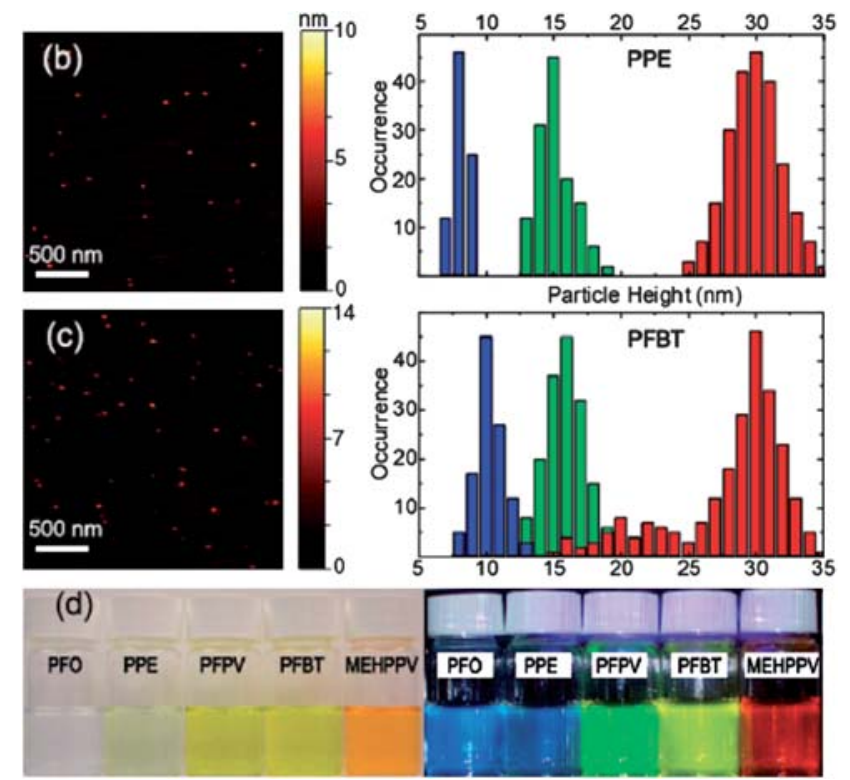

Fig. 5 (b) Typical the atomic force microscopy (AFM) image of small PPE dots and histograms of particle height of the PPE dots prepared with different precursor concentration; (c) typical AFM image of small PFBT dots and histograms of particle height of the PFBT dots prepared with different precursor concentrations; (d) photographs of aqueous CPNs suspensions under room light (left) and UV light (right) illumination. Reprinted with permission from ref. 11 (Copyright 2008 The American Chemical Society). between the donor and acceptor molecules and for an efficient energy transfer the emission spectrum of the energy donor should overlap with the absorption spectrum of the energy acceptor. ${ }^{34}$

Energy transfer has been successfully applied in conjugated polymer blends to enhance the quantum efficiency and to tune the emission colour of the light emitting devices. ${ }^{35-37}$ There are also a good number of examples of energy transfer-facilitated sensor applications such as DNA and protein sensing. ${ }^{38-40}$ Biological sensor applications require the use of water-soluble materials. To this end, water dispersible conjugated polymer nanoparticles are suitable for biological applications. Moreover, high absorption cross-sections and high sensitivity for the excitation light due to mobile excitons associated with these nanoparticles make them a valuable model for studying energy transfer in complex nanoscale systems. This model would help us to understand the underlying photophysics in such systems and this in turn would be of benefit for enhancing the performance of nanoparticle-based sensing schemes and nanostructured electroluminescent device layers.

In 2006, first dye-doped conjugated polymer nanoparticles were prepared by Grigalevicius and co-workers and the excitation energy transfer from the excited chromophor of the nanoparticle to the fluorescent dye was demonstrated. ${ }^{41}$ For this purpose, negatively charged nanoparticles were prepared from a blue emitting polyfluorene derivatives by using the anionic surfactant sodium dodecyl sulfate during the miniemulsion process and incorporating two cationic fluorescent dyes such as Rhodamine $6 \mathrm{G}$ and tetramethylrhodamine ethyl ester perchlorate (Rhodamine TM). The results showed that the blue fluorescence of the dye-coated polyfluorene nanoparticles was slightly quenched after doping with the dyes and a new emission band of the surface-bound dye (Rhodamine $6 \mathrm{G}$ or Rhodamine TM) appeared in the wavelength region of 530-600 nm indicating an effective excitation energy transfer from the excited PF chromophores to the fluorescent dye.

In the same year, McNeill and co-workers synthesised nanoparticles using the reprecipitation method from the blue-emitting conjugated polymer polyfluorene (PDHF) doped with varying amounts of green-, yellow-, and red-emitting conjugated polymers (PFPV, PFBT, MEH-PPV, respectively, see structures in Fig. 6) and studied energy transfer from host polymer to dopant polymers. ${ }^{12}$ They described the nanoparticles as a system of densely packed chromophores exhibiting efficient energy transfer from the host to the dopant polymers. A particle height histogram obtained from the atomic force microscopy (AFM) images indicates that most of the nanoparticles have diameters in the range of 20-30 nm. They estimated 100-200 molecules per nanoparticle by 


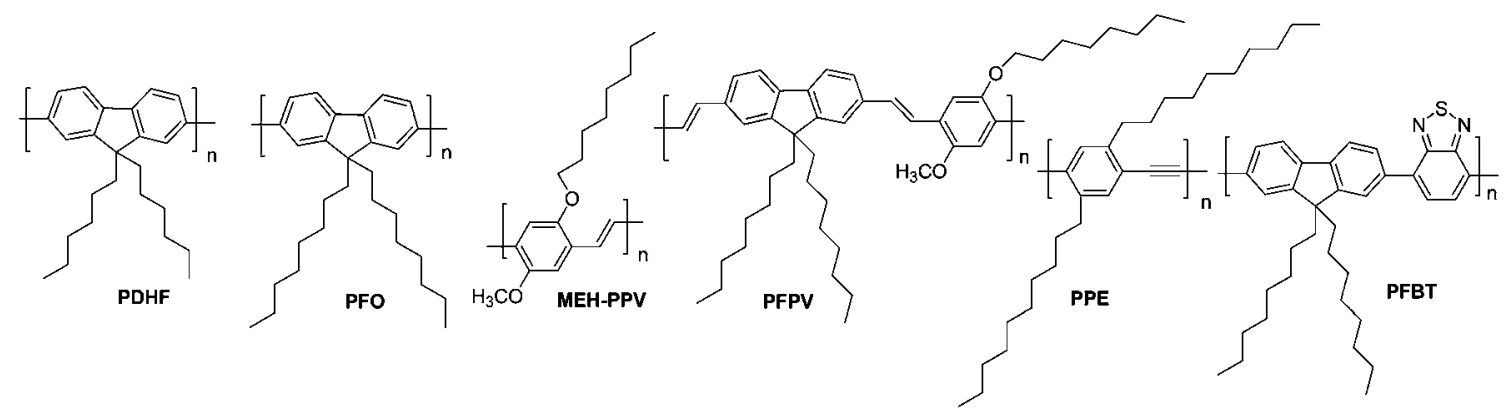

Fig. 6 The structures of poly(9,9-dihexylfluorene) (PDHF), poly(9,9-dioctylfluorene) (PFO), poly[2-methoxy-5-(2-ethylhexyloxy)-1,4-phenylenevinylene] (MEH-PPV), poly[2-methoxy-5-(2-ethylhexyloxy)-2,7-(9,9-dioctylfluorene)] (PFPV), poly(p-phenylene ethynylene) (PPE), poly(9,9-dioctylfluorene-2,7-diyl-co-benzothiadiazole) (PFBT).

assuming that these were densely packed spherical particles. Apparently, particle size and morphology were not affected by the presence of a dopant. The fluorescence spectra of nanoparticles with varying amounts of dopant concentration (up to $10 \mathrm{wt} \%$ ) are shown in Fig. 7. As can be seen, PF emission decreases with increasing dopant concentration while the emission intensity of dopants increases and reaches a maximum around $6 \mathrm{wt} \%$. However, the dopant concentration higher than $6 \%$ causes a decrease in the fluorescent quantum yield.

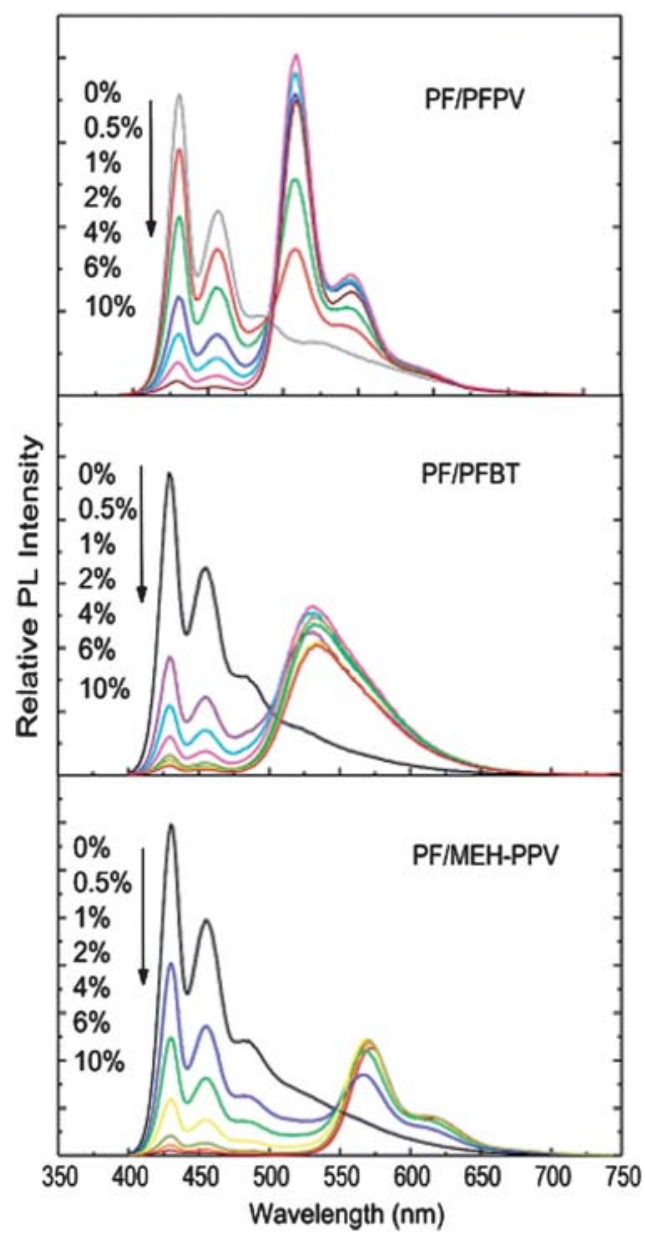

The dopant (acceptor) concentration-dependent fluorescence quenching of the host polymer was studied using the SternVolmer relation. However, they observed a significant deviation from the Stern-Volmer relation in the composition-dependent nanoparticle fluorescence in some cases. To explain this, they developed a nanoparticle energy transfer model by taking into account statistical variability in nanoparticle composition. This model provided a good agreement with experimental results, particularly for the dopants with high quenching efficiencies. The

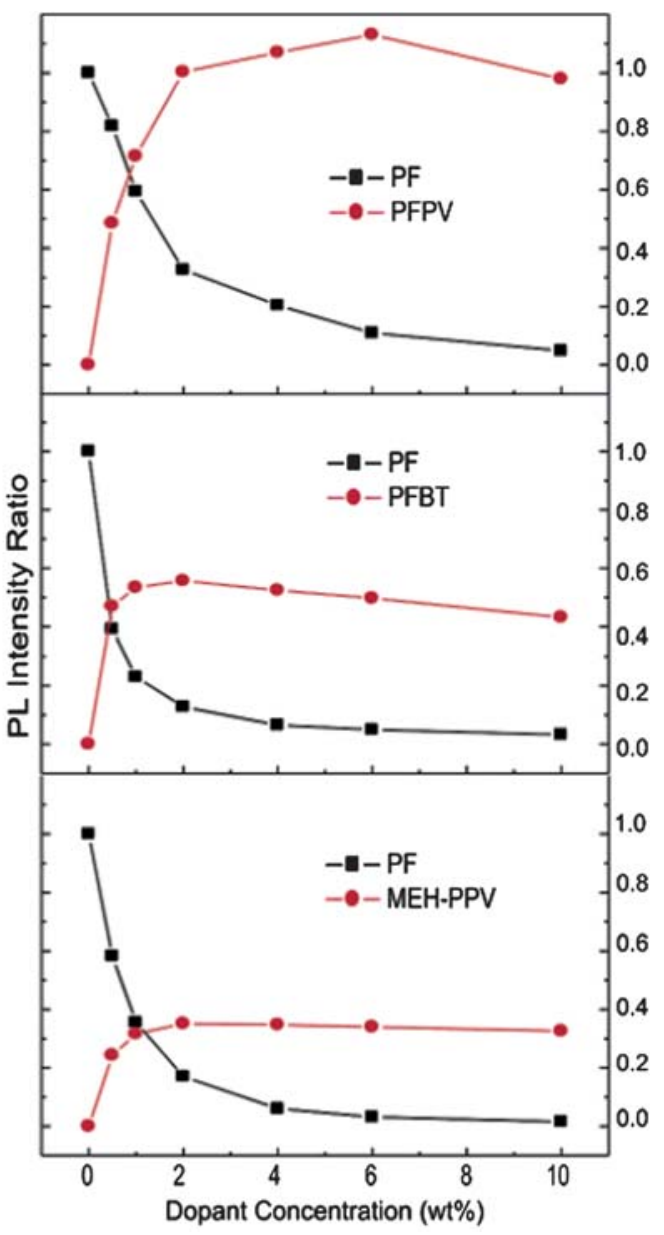

Fig. 7 (Left) Concentration-dependent fluorescence spectra of polymer blend nanoparticles under 375 nm excitation. (Right) Fluorescence intensity change of PF host and dopant polymers as a function of dopant concentration in blend nanoparticles. All fluorescence emission intensities were normalized to the $430 \mathrm{~nm}$ emission of pure PF nanoparticles. Reprinted with permission from ref. 12 (Copyright 2008 The American Chemical Society). 
results obtained from both Stern-Volmer relation and the nanoparticle energy transfer model indicated highly efficient energy transfer between the donor (host polymer) and the acceptor (dopant polymers) molecules. Both models suggest that approximately 100 or more host molecules are quenched by a single dopant molecule. They interpreted this result as evidence of long-range $(>10 \mathrm{~nm})$ energy transport by multiple energy transfer. Taking into account the molar absorptivity and quantum yield, the fluorescence brightness of the blend nanoparticles was estimated to be approximately 100 times larger than that of quantum dots, and 20 times higher than that of dyeloaded silica spheres of similar dimensions. ${ }^{12}$

In 2008, McNeill and co-workers prepared polyfluorene based nanoparticles doped with various dyes such as perylene, tetraphenylporphyrin, coumerin 6 and nile red using the reprecipitation method. They studied the energy transfer by steady-state and time-resolved spectroscopy and compared their experimental results to the model which was developed by taking into consideration the combined effect of Förster energy transfer, energy diffusion and particle size. The measurement results indicated very efficient energy transfer from host (donor) polyfluorene to dopant (acceptor) dye molecules. For aqueous suspensions of $\sim 30 \mathrm{~nm}$ diameter polymer nanoparticles doped with perylene or coumarin $6(2 \mathrm{wt} \%)$, the fluorescence quantum yield and peak extinction coefficients were measured to be $\sim 40 \%$ and $1.5 \times 10^{9} \mathrm{M}^{-1} \mathrm{~cm}^{-1}$, respectively. They also conducted photobleaching experiments: the results indicated that the photostability of the conjugated polymers could be improved by doping them with energy acceptors. In this detailed study, it was shown that these nanoparticles had indeed distinctive features such as high brightness, highly red-shifted emission spectrum, and excellent photostability. ${ }^{13,42}$

\section{Optoelectronic applications}

Nanoparticles made using the miniemulsion method have been extensively studied and used in optoelectronic device fabrication by Scherf, Landfester, List, and co-workers, who reported monolayer polymer light emitting diodes (PLEDs), multilayer PLEDs, light-emitting electrochemical cells (LECs) and solar cells. $^{43-49}$

Although the authors have shown that photophysical and electroluminescent properties of the conjugated polymers were not influenced upon the nanoparticle formation via the miniemulsification process, it would be still interesting to fabricate optoelectronic devices from small, single polymer chain containing nanoparticles prepared via the reprecipitation method in which there would be no additives including surfactants, stabilizing agents and hydrophobes. However, there are no examples yet of devices fabricated from these nanoparticles. This particular feature is waiting to be explored.

Here, we would like to provide various examples for the optoelectronic applications of nanoparticles with sizes ranging from 50-500 $\mathrm{nm}$ made via the miniemulsion method. For example, such a device that contains a homogeneous single layer of nanoparticles based on a methyl-substituted ladder-type poly(p-phenylene) (m-LPPP) is a light emitting diode. This nanoparticle based LED is reported to exhibit enhanced optoelectronic characteristics (with a lower onset and slightly higher efficiency) compared with OLED devices fabricated directly from the conjugated polymer films. The authors attributed this behaviour to an enhanced electron injection from cathode as a consequence of in situ formation of a 'stalactite'-type nanostructured cathode during aluminium evaporation. Optical spectroscopy has evidenced that conjugated polymers can be converted into aqueous nanoparticle dispersions without generation of electronic defects; the nanoparticles display the photophysical properties of the bulk polymer. ${ }^{43}$

To demonstrate the use of nanoparticles in the solar cell devices, the nanoparticles with mean diameters of approximately $50 \mathrm{~nm}$, containing either the pure polymer components hole accepting polymer, PFB [poly(9,9-dioctylfluorene-2,7-diyl-cobis- $N, N^{\prime}$-(4-butylphenyl)-bis- $N, N^{\prime}$-phenyl-1,4-phenylenediamine)], and electron accepting polymer, PFBT [poly(9,9-dioctylfluorene2,7-diyl-co-benzothiadiazole)], or a mixture of PFB and PFBT in each particle were prepared. Photovoltaic devices based on these particles have been studied with respect to the correlation between external quantum efficiency and layer composition. The properties of devices containing a blend of single-component PFB and PFBT particles were shown to differ significantly from those of solar cells based on blend particles, even for the same layer composition. An external quantum efficiency of approximately $4 \%$ is measured for a device made from polymer blend nanoparticles containing PFB:F8BT at a weight ratio of $1: 2$ in each individual nanosphere. ${ }^{44,45}$

These nanoparticles were also utilized to form organic multilayer structures and devices. In one study, one layer was made of miniemulsion processed nanoparticles and the other layer was spin-coated using a regular conjugated polymer dissolved in common organic solvents. Conjugated polymers grafted with hydrophilic side chains were found to improve the wetting and film forming properties of the semiconducting polymer nanospheres deposited from aqueous dispersion. These solution processed conjugated polymer multilayer structures were reported to hold promise for light emitting devices. ${ }^{46}$

The same group has also reported on the fabrication of PLED and light-emitting electrochemical cells (LECs) in planar surface cell geometry (with the anode and the cathode made of gold having an interelectrode spacing of $1 \mu \mathrm{m}$ ) by means of inkjet printing. The active material for PLED was an aqueous MEHPPV dispersion, and for LECs their blends with poly(ethylene oxide) and lithium triflate formed the solid state electrolyte. ${ }^{47}$

In a recent work of List and co-workers it was demonstrated that an aqueous dispersion of conjugated polymer nanoparticles could be coated by inkjet printing onto a polymer surface patterned by soft embossing as shown in Fig. 8. In this study, first a polymeric template from polystyrene was prepared using a stamp of poly(dimethylsiloxane) (PDMS) and then the dispersion containing the nanoparticles was inkjet printed onto this surface. The nanoparticles were self-assembled in the grooves of the template. They incorporated both the template layer and the assembled nanoparticles into a device structure and demonstrated its use in polymer light emitting devices. ${ }^{49}$

\section{Biological and biomedical applications of CPNs}

The CPNs used in the previous examples are made from hydrophobic polymers. However, for biomedical applications it 


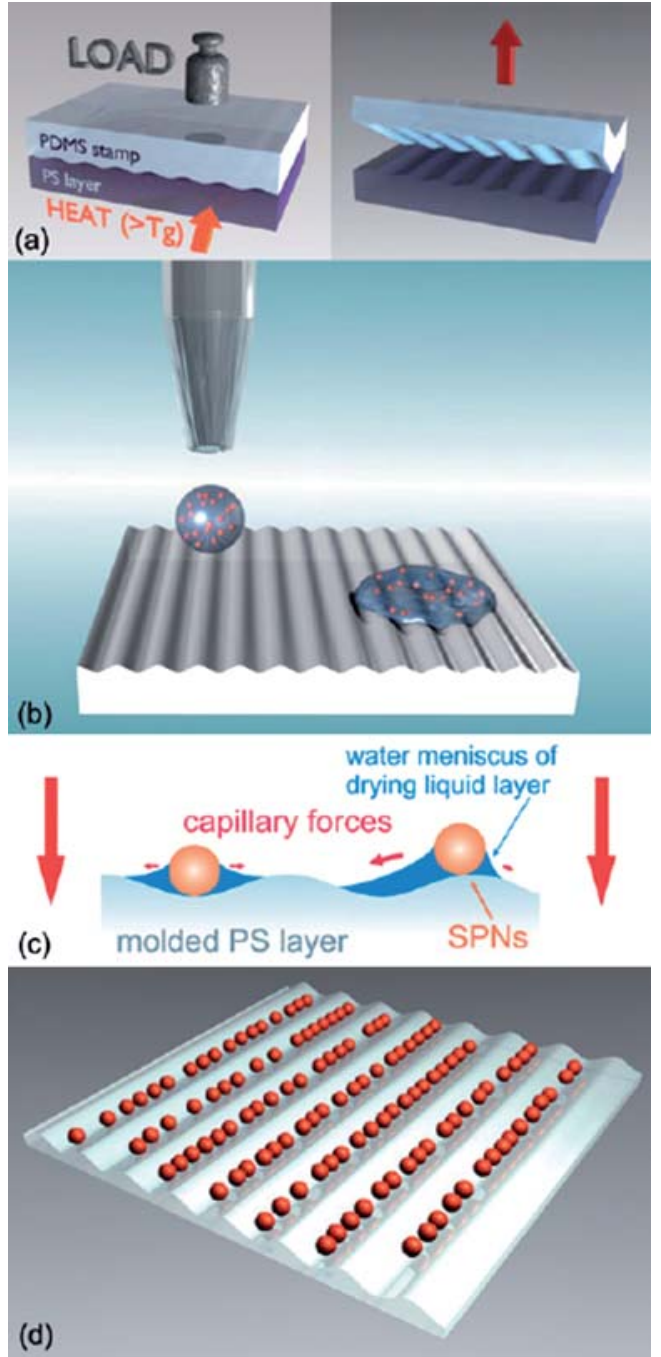

Fig. 8 Schematic of the fabrication process for functional nanostructures from assembled conjugated polymer nanoparticles. First a polymeric template is fabricated by soft embossing with a PDMS stamp (a); then the dispersion containing the nanoparticles is inkjet printed onto this surface (b). Due to capillary forces acting on the spheres (c) they finally assemble in the grooves of the template while the drop dries (d). Reprinted with permission from ref. 49 (Copyright 2009 The Royal Society of Chemistry).

would be more appropriate to utilize nanoparticles having hydrophilic surface groups to prevent aggregation in long term imaging.

For this purpose, recently Moon and co-workers synthesized CPNs based on amine containing PPE by ultrafiltration and demonstrated the use of CPNs for $2 \mathrm{P}$ imaging of endothelial cells in a tissue model. ${ }^{20}$ It was shown that the size of nanoparticles could be tuned depending on the counter ions selected. They measured the 2P-action cross section of CPNs between 1000 $11000 \mathrm{GM}$ with a maximum of about $730 \mathrm{~nm}$, which is a few orders of magnitude higher than the value for the current organic fluorophores and close to the values of quantum dots (QDs). ${ }^{41,42}$ The average lifetimes of CPNs were measured using fluorescence lifetime microscopy and found 10-50 times faster than QDs, corresponding to a higher photon emission rate, $\mathrm{CPNs}$ are brighter than QDs. The photostability of these nanoparticles were also studied by incubating live BALB/C3T3 fibroblasts with CPN and QD525 (Qtracker 525, Invitrogen) respectively. Cells were fixed with paraformaldehyde and samples were continuously imaged at $780 \mathrm{~nm}$ with a power of $2 \mathrm{~mW}$ for a $1 \mathrm{~h}$ period. During this period, similar results were obtained in CPN and QD indicating high resistance to bleaching in CPNs, which is comparable to that of QDs. The lack of toxic effects during longterm monitoring of capillary formation in a microfluidic device also supports the potential of CPNs for in vitro and possibly in vivo imaging applications. ${ }^{20}$

Water-dispersible CPNs were prepared using an amine containing poly(p-phenylene ethynylene) (PPE) to demonstrate livecell imaging. Various cells, including baby-hamster kidney (BHK) and BALB/C 3T3 (mouse embryonic fibroblast), were incubated with CPNs in culture media for various time periods (from one hour to several days) to examine the cell permeability, photostability, and cellular toxicity of CPNs. Fig. 9 shows microscopic images of live BALB/C (a) and fixed $3 \mathrm{~T} 3$ cells (b) stained by the CPNs overnight. The results showed that the CPNs were cell permeable and accumulate exclusively in the cytosol without any considerable inhibition of cell viability. In addition, it was demonstrated that CPNs exhibit high resistance to photobleaching, in contrast to commercially available dyes. ${ }^{50}$

Recently Green and co-workers prepared CPNs with an average particle diameter of $13 \mathrm{~nm}$ from poly[2-( $\left(2^{\prime}, 5^{\prime}-\operatorname{bis}\left(2^{\prime \prime}-\right.\right.$ ethylhexyloxy)phenyl)-1,4-phenylene vinylene] (BEHP-PPV) capped with poly(ethylene glycol) (PEG) by a miniemulsion route and used these nanoparticles in the intracellular imaging experiments. ${ }^{51}$ Hep-2 cells were incubated with BEHP-PPV particles for $24 \mathrm{~h}$. The particles mainly concentrated in the cytoplasm and only some of them passed into nuclei apparently through the nuclear pore complexes by passive diffusion. Through a series of control experiments, they demonstrated that these nanoparticles are not cytotoxic and do not show significant photobleaching over a time period of approximately 5 min when using a $405 \mathrm{~nm}, 25 \mathrm{~mW}$ diode laser at 50\% and taking images every $1.635 \mathrm{~s}$. Another interesting finding was that when BEHPPPV particles labelled cells stored in $70 \% \mathrm{MeOH}$ and $30 \%$ acetone mixture for a month, they preserved their stability well
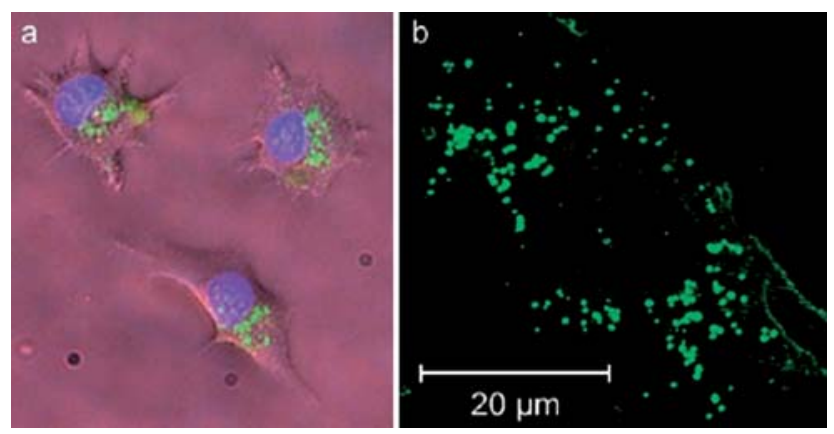

Fig. 9 Fluorescence images of live (a) and fixed (b) cells. (a) BALB/C $3 \mathrm{~T} 3$ cells were incubated sequentially with CPNs (green) and Hoechst dye (blue). The image is a composite of two micrographs using GFP (for CPNs) and DAPI/Hoechst/AMCA (for Hoechst) filter sets. (b) Live BALB/C 3 T3 cells were incubated with CPNs and fixed for confocal microscopic study. Reproduced with permission from ref. 50 (Copyright 2007 Wiley-VCH Verlag GmbH \& Co. KGaA). 
and no quenching in the fluorescent emission compared to initial emission intensity was observed. ${ }^{51}$

In another work, Green and co-workers synthesised CPNs based on MEH-PPV by the miniemulsion method using sodium dodecylsulfate (SDS) and PEG as surfactants. SDS-capped nanoparticles were incubated with CHO-K1 (Chinese Hamster ovary) cells and found to be toxic to the cells. However, they show good photostability. When PEG-capped MEH-PPV nanoparticles were incubated with h-TERT human fibroblast cells, the particles were observed inside cells and around the nuclear area. The preliminary results indicate that these nanoparticles are not cytotoxic. ${ }^{52}$

Screening the activity of protein kinases can provide an understanding of many fundamental biological processes and be valuable for the diagnosis of various diseases. Moon and coworkers designed and synthesized CPNs for biochemical protein kinase assay based on fluorescence energy transfer. Nanoparticles were prepared from a derivative of poly( $p$-phenylene ethynylene) containing pentiptycene and a monomer with iminodiacetic acid. These nanoparticles were treated with $\mathrm{GaCl}_{3}$
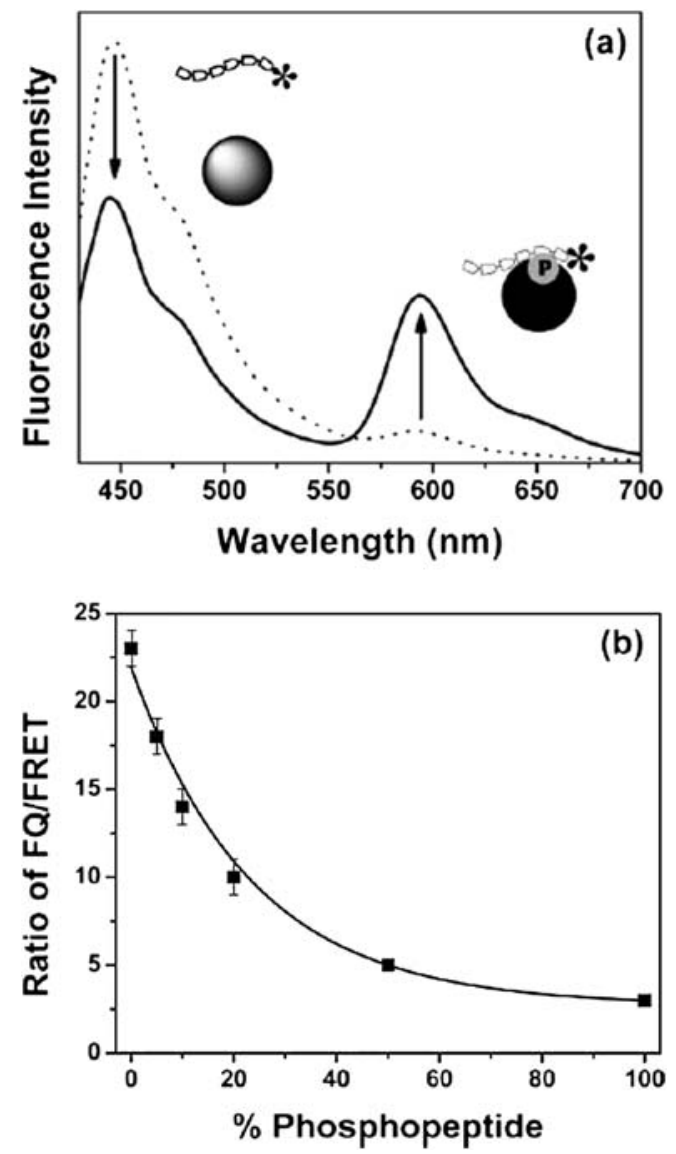

Fig. 10 (a) Emission spectra of CPN mixed with rhodamine-labeled kemptide (Rh-kemptide, dotted line) and with rhodamine-labeled phosphokemptide (Rh-p-kemptide, solid line). Strong FRET occurred from metal ion-assisted adsorption of phosphokemptides, while no significant FRET was observed from nonphosphorylated kemptide. (b) Ratiometric (fluorescence quenching (FQ)/FRET) calibration curve generated from standard mixtures of Rh-kemptide and Rh-p-kemptide. Reprinted with permission from ref. 19 (Copyright 2007 The Royal Society of Chemistry). to allow chelating with iminoacetic acid group onto CPNs for the selective detection of phosphopeptides. When CPNs/Ga solution was mixed with rhodamine-labelled kemptide (Rh-kemptide), no energy transfer was observed, while an efficient energy transfer was achieved by mixing rhodamine-labelled phosphokemptide (Rh-p-kemptide) with CPNs/Ga (Fig. 10a). They observed that the fluorescence quenching and FRET were proportional to the amount of phosphorylation (Fig. 10b). They also monitored the kinase activity as a function of the inhibitor by incubating Rhkemptide with protein kinase $\mathrm{A}$ in the presence of adenosine triphosphate (ATP). In the presence of CPNs/Ga the degree of phosphorylation was measured by calculating the ratio between the fluorescence quenching of the CPNs at $455 \mathrm{~nm}$ and the FRET signal at $585 \mathrm{~nm} .{ }^{19}$

Very recently, McNeill and co-workers demonstrated the use of CPNs in the energy-transfer-mediated phosphorescence from metalloporphyrin-doped polyfluorene nanoparticles and its application to biological oxygen sensing. ${ }^{53}$ In this work, poly $(9,9-$ dihexylfluorene) (PDHF) and poly(9,9-dioctylfluorene) (PFO) were chosen as the host (donor) and platinum(II) octaethylporphine (PtOEP) was utilized as the oxygen sensitive dye (acceptor) (Fig. 11). Highly efficient energy transfer from the donor polymer (PFHF or PFO) to acceptor dye (PtOEP) was observed by fluorescence spectroscopy and time resolved measurements. This efficient energy transfer resulted in molecular oxygen-sensitive phosphorescence. A dispersion of nitrogen-saturated CPNs was excited by using a UV lamp $(365 \mathrm{~nm})$. The bright phosphorescence was observed to fade within a few seconds upon exposure to air indicating the oxygen permeability. In order to understand whether this event is $\mathrm{pH}$-sensitive, fluorescence spectra were recorded in a series of phosphate buffers with $\mathrm{pH}$ values ranging

a)

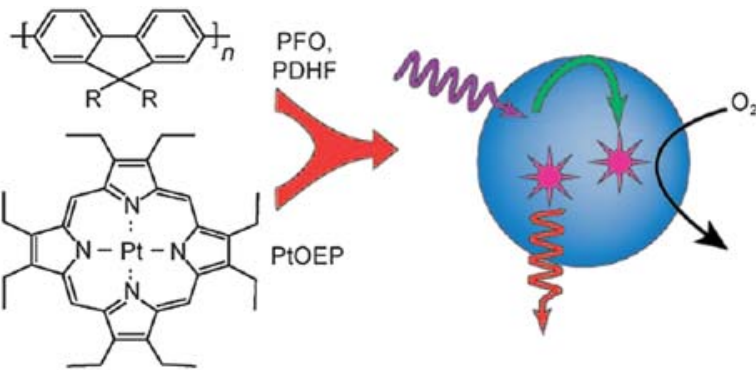

b)

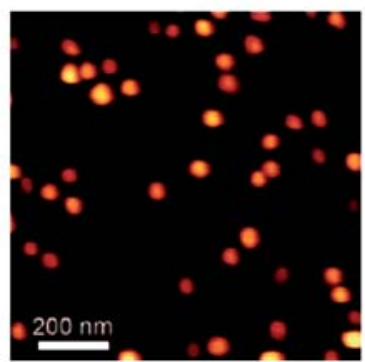

c)

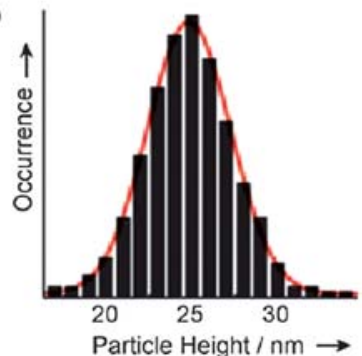

Fig. 11 a) Schematic illustration of the formation of conjugated polymer dots for oxygen sensing. b) Representative AFM image of PtOEPdoped PDHF dots dispersed on a mica substrate. c) Histogram of particle-height data obtained from the AFM image in (b). Reproduced with permission from ref. 53 (Copyright 2009 Wiley-VCH Verlag GmbH \& Co. KGaA). 
from 5 to 8; however, the results showed no apparent sensitivity to $\mathrm{pH}$ over this range. As a further test of the brightness and sensing capabilities of the CPNs, single-particle phosphorescence imaging was performed. Single-particle phosphorescence imaging results indicated the observed phosphorescence intensity from single particles was sensitive to the partial pressure of oxygen. To understand the cellular uptake of CPNs, a macrophage-like murine cell line was incubated with these doped nanoparticles for $10 \mathrm{~h}$ in media. The differential interference contrast and phosphorescence images showed uptake of the nanoparticle sensor by macrophage cells. The results also indicated that these nanoparticles did not appear to exhibit cytotoxicity or phototoxicity.

\section{Conclusions and outlook}

Nanoparticles based on conjugated polymers are highly versatile nano-structured materials. They can find applications in various areas such as optoelectronics, photonics, bio-imaging, biosensing and nanomedicine owing to their straightforward synthesis in desired sizes and properties, biocompatibility and inherent non-toxicity.

A number of different conjugated polymers have been used for the formation of CPNs. Although nanoparticles with hydrophilic surface functional groups which can limit the intrinsic hydrophobicity of the CPNs are highly desirable in biological applications, the literature examples are mostly limited to highly hydrophobic conjugated polymers carrying no functional groups to be further modified. However, we believe that this particular problem can be overcome by designing and synthesising new generation CPNs.

There has also been little effort in developing stable CPNs without using surfactants and hydrophobes and exploring their applications in the area of optoelectronics. Another drawback preventing the exploitation of CPNs is the mechanical instability of these nanoparticles. To this end, the development of CPNs which can be mechanically stable in water as well as in organic solvents and the surface functionalization of these nanoparticles could be highly valuable for many applications.

\section{Acknowledgements}

Support by EU-UNAM-REGPOT Grant No:203953 and ESFEURYI is gratefully acknowledged.

\section{References}

1 (a) K. Landfester, Angew. Chem., Int. Ed., 2009, 48, 4488; (b)

K. Landfester, Annu. Rev. Mater. Res., 2006, 36, 231.

2 K. Landfester, Adv. Mater., 2001, 13, 765.

3 K. Landfester, Macromol. Rapid Commun., 2001, 22, 896.

4 K. Landfester, R. Montenegro, U. Scherf, R. Güntner, U. Asawapirom, S. Patil, D. Neher and T. Kietzke, Adv. Mater., 2002, 14, 651.

5 P. Sarrazin, D. Chaussy, L. Vurth, O. Stephan and D. Beneventi, Langmuir, 2009, 25(12), 6745.

6 K. Müller, M. Klapper and K. Müllen, Macromol. Rapid Commun., 2006, 27, 586.

7 A. Berkefeld and S. Mecking, Angew. Chem., Int. Ed., 2006, 45, 6044.

8 M. C. Baier, J. Huber and S. Mecking, J. Am. Chem. Soc., 2009, 131, 14267.

9 C. Wu, C. Szymanski and J. McNeill, Langmuir, 2006, 22, 2956.
10 C. Wu, C. Szymanski, Z. Cain and J. McNeill, J. Am. Chem. Soc., 2007, 129, 12904.

11 C. Wu, B. Bull, C. Szymanski, K. Christensen and J. McNeill, ACS Nano, 2008, 2, 2415.

12 C. Wu, H. Peng, Y. Jiang and J. McNeill, J. Phys. Chem. B, 2006, 110, 14148.

13 C. Wu, Y. Zheng, C. Szymanski and J. McNeill, J. Phys. Chem. C, 2008, 112, 1772.

14 C. Wu and J. McNeill, Langmuir, 2008, 24, 5855.

15 H. Peng, C. Wu, Y. Jiang, S. Huang and J. McNeill, Langmuir, 2007, 23, 1591 .

16 C. Szymanski, C. Wu, J. Hooper, M. A. Salazar, A. Perdomo, A. Dukes and J. McNeill, J. Phys. Chem. B, 2005, 109, 8543.

17 N. Kurokawa, H. Yoshikawa, N. Hirota, K. Hyodo and H. Masuhara, ChemPhysChem, 2004, 5, 1609.

18 J. H. Moon, R. Deans, E. Krueger and L. F. Hancock, Chem. Commun., 2003, 104.

19 J. H. Moon, P. MacLean, W. McDaniel and L. F. Hancock, Chem. Commun., 2007, 4910.

20 N. A. A. Rahim, W. McDaniel, K. Bardon, S. Srinivasan, V. Vickerman, P. T. C. So and J. H. Moon, Adv. Mater., 2009, 21, 3492.

21 T. Piok, C. Gadermaier, F. P. Wenzl, S. Patil, R. Montenegro, K. Landfester, G. Lanzani, G. Cerullo, U. Scherf and E. J. W. List, Chem. Phys. Lett., 2004, 389, 7.

22 (a) I. O. Huyal, T. Ozel, D. Tuncel and H. V. Demir, Opt. Express, 2008, 16, 13391; (b) I. O. Ozel, T. Ozel, H. V. Demir and D. Tuncel, Opt. Express, 2010, 18, 670.

23 J. K. Grey, D. Y. Kim, B. C. Norris, W. L. Miller and P. F. Barbara, J. Phys. Chem. B, 2006, 110, 25568.

24 S. J. Park, A. J. Gesquiere, J. Yu and P. F. Barbara, J. Am. Chem. Soc., 2004, 126, 4116.

25 X. Michalet, F. F. Pinaud, L. A. Bentolila, J. M. Tsay, S. Doose, J. J. Li, G. Sundaresan, A. M. Wu, S. S. Gambhir and S. Weiss, Science, 2005, 307, 538.

26 X. Gao, Y. Cui, R. M. Levenson, L. W. K. Chung and S. Nie, Nat. Biotechnol., 2004, 22, 969.

27 K.-Y. Pu and B. Liu, Biosens. Bioelectron., 2009, 24, 1067.

28 D. T. McQuade, A. E. Pullen and T. M. Swager, Chem. Rev., 2000, 100, 2537.

29 K. Dore, S. Dubus, H. A. Ho, I. Levesque, M. Brunette, G. Corbeil, M. Boissinot, G. Boivin, M. G. Bergeron, D. Boudreau and M. Leclerc, J. Am. Chem. Soc., 2004, 126, 4240.

30 B. Liu, T. T. T. Dan and G. C. Bazan, Adv. Funct. Mater., 2007, 17, 2432.

31 O. R. Miranda, C. C. You, R. Phillips, I. B. Kim, P. S. Ghosh, U. H. F. Bunz and V. M. Rotello, J. Am. Chem. Soc., 2007, 129, 9856.

32 M. D. Disney, J. Zheng, T. M. Swager and P. H. Seeberger, J. Am. Chem. Soc., 2004, 126, 13343.

33 F. Helmchen and W. Denk, Nat. Methods, 2005, 2, 932.

34 J. R. Lakowicz, Principles of Fluorescence Spectroscopy, 3rd ed., Springer, Berlin, 2006.

35 C. F. Huebner, R. D. Roeder and S. H. Foulger, Adv. Funct. Mater., $2009, \mathbf{1 9}, 1$.

36 J. Luo, X. Li, Q. Hou, J. Peng, W. Yang and Y. Cao, Adv. Mater., 2007, 19, 1113.

37 J.-I. Lee, I.-N. Kang, D.-H. Hwang, H.-Ku Shim, S. C. Jeoung and D. Kim, Chem. Mater., 1996, 8, 1925.

38 J. Liu, Z. Xie, Y. Cheng, Y. Geng, L. Wang, X. Jing and F. Wang, Adv. Mater., 2007, 19, 531.

39 L. Chen, D. W. McBranch, H. L. Wang, R. Helgeson, F. Wudl and D. G. Whitten, Proc. Natl. Acad. Sci. U. S. A., 1999, 96, 12287.

40 C. H. Fan, S. Wang, J. W. Hong, G. C. Bazan, K. W. Plaxco and A. J. Heeger, Proc. Natl. Acad. Sci. U. S. A., 2003, 100, 6297.

41 S. Grigalevicius, M. Forster, S. Ellinger, K. Landfester and U. Scherf, Macromol. Rapid Commun., 2006, 27, 200.

42 E. J. Harbron, C. M. Davis, J. K. Campbell, R. M. Allred, M. T. Kovary and N. J. Economou, J. Phys. Chem. C, 2009, 113, 13707.

43 T. Piok, S. Gamerith, C. Gadermaier, H. Plank, F. P. Wenzl, S. Patil, R. Montenegro, T. Kietzke, D. Nehrer, U. Scherf, K. Landfester and E. J. W. List, Adv. Mater., 2003, 15, 800.

44 T. Kietzke, D. Nehrer, K. Landfester, R. Montenegro, R. Güntner and U. Scherf, Nat. Mater., 2003, 2, 408. 
45 T. Kietzke, D. Nehrer, M. Kumke, R. Montenegro, K. Landfester and U. Scherf, Macromolecules, 2004, 37, 4882.

46 T. Piok, H. Plank, G. Mauthner, S. Gamerith, C. Gadermaier, F. P. Wenzl, S. Patil, R. Montenegro, M. Bouguettaya, J. R. Reynolds, U. Scherf, K. Landfester and E. J. W. List, Jpn. J. Appl. Phys., 2005, 44, 479.

47 G. Mauthner, K. Landfester, A. Köck, H. Brückl, M. Kast, C. Stepper and E. J. W. List, Org. Electron., 2008, 9, 164.

48 E. Fisslthaler, S. Sax, U. Scherf, G. Mauthner, E. Moderegger, K. Landfester and E. J. W. List, Appl. Phys. Lett., 2008, 92, 183305.
49 E. Fisslthaler, A. Blümel, K. Landfester, U. Scherf and E. J. W. List, Soft Matter, 2008, 4, 2448.

50 J. H. Moon, W. McDaniel, P. MacLean and L. E. Hancock, Angew. Chem., Int. Ed., 2007, 46, 8223.

51 P. Howes, R. Thorogate, M. Green, S. Jickells and B. Daniel, Chem. Commun., 2009, 2490.

52 M. Green, P. Howes, C. Berry, O. Argyros and M. Thanou, Proc. R. Soc. London, Ser. A, 2009, 465, 2751.

53 C. Wu, B. Bull, K. Christensen and J. McNeill, Angew. Chem., Int. Ed., 2009, 48, 2741. 